\title{
A Scoping Review of Homebound Older People: Definition, Measurement and Determinants
}

\author{
Young Ko (D) and Wonjung Noh * (D) \\ College of Nursing, Gachon University, Incheon 21936, Korea; moodory@gmail.com \\ * Correspondence: wjnoh@gachon.ac.kr; Tel.: +82-32-820-4228
}

Citation: Ko, Y.; Noh, W. A Scoping Review of Homebound Older People: Definition, Measurement and Determinants. Int. J. Environ. Res. Public Health 2021, 18, 3949. https:// doi.org/10.3390/ijerph18083949

Academic Editor: Paul B. Tchounwou

Received: 17 March 2021

Accepted: 7 April 2021

Published: 9 April 2021

Publisher's Note: MDPI stays neutral with regard to jurisdictional claims in published maps and institutional affiliations.

Copyright: (c) 2021 by the authors. Licensee MDPI, Basel, Switzerland. This article is an open access article distributed under the terms and conditions of the Creative Commons Attribution (CC BY) license (https:// creativecommons.org/licenses/by/ $4.0 /)$.

\begin{abstract}
Being homebound (HB) can affect people's physical and mental health by decreasing movement, which can itself be exacerbated by the deterioration of people's health. To break this vicious cycle of $\mathrm{HB}$ and being in poor health, it is necessary to identify and address the factors influencing HB status. Thus, we used a scoping review to identify an HB trend, focusing on the definition, measurements, and determinants of HB status. We analyzed 47 studies according to the five-stage methodological framework for scoping reviews. The common attribute of definitions of $\mathrm{HB}$ status was that the boundaries of daily life are limited to the home. However, this varied according to duration and causes of becoming HB; thus, the understanding of HB shifted from the presence or absence of being HB to the continuum of daily activity. Various definitions and measurements have been used to date. Many studies have focused on individual factors to analyze the effect of HB. In the future, it will be necessary to develop a standardized measurement that reflects the multidimensional $\mathrm{HB}$ state. In addition, it is necessary to utilize a theoretical framework to explore the social and environmental factors affecting HB.
\end{abstract}

Keywords: older adults; homebound; determinants; definition; scoping review

\section{Introduction}

The number and proportion of people aged 60 years or older is rapidly increasing worldwide. According to the World Health Organization, there were one billion people aged 60 or older in 2019. The number of older people is expected to increase to 1.4 billion by 2030 and 2.1 billion by 2050 [1]. This increase in the older population can also lead to an increase in the proportion of homebound (HB) people who stay at home [2].

HB people suffer from multiple physical health problems, such as metabolic, cardiovascular, cerebrovascular, and musculoskeletal diseases, as well as psychiatric health problems, such as cognitive impairment, dementia, and depression [3]. Despite the high demand for medical care services, their access to healthcare services is limited because of their physical and economic dependency coupled with a lack of information and support [4]. In addition, because they are noncompliant with medication adherence and care pattern rules, they use healthcare services more and spend more on health management than those who are not HB [5]. Existing social networks may be reduced or eliminated because they are HB [6]. Loneliness may arise when there is a mismatch between desired and available forms of social and emotional connections [7]. Therefore, HB status is a possible risk factor that increases social isolation and loneliness. Eventually, HB leads to negative health outcomes such as mortality [8,9] and a low quality of life [10]. HB adversely affects health [8], and when health deteriorates, it causes HB again [11]. To avoid this vicious cycle of $\mathrm{HB}$ and poor health, it is necessary to explore the causes and status of $\mathrm{HB}$.

Several countries have services to manage HB. In the United States, skilled nursing care, physical therapy, speech-language pathology services, and continued occupational services have been provided to HB people [12]. Singapore also provides home-based services such as medical care, nursing care, personal care, therapy, palliative care, meals on wheels, and transport/escort services to the HB elderly [13]. In European countries, 
a proportion of older adults, including frail and ill older adults, receive home care [14]. For example, Sweden provides primary health care by a physician and visiting nurse and home help, including social care and personal care, to older adults staying in their own homes [15].

However, these services have only focused on solving problems caused by $\mathrm{HB}$, and there is a lack of preventative services. In Japan, the focus was initially on troubleshooting $\mathrm{HB}$, but it was changed to focus on prevention because continuous service, which focused on problem solving, was recognized as limited [16]. Therefore, it is important to understand the current situation, as well as interventions for older adults with $\mathrm{HB}$ and prevention of HB among older adults.

Studies on the prevalence of $\mathrm{HB}$ and its influencing factors have been conducted since the 1990s [17,18]. In an early study, HB was defined as being confined to the home [17]. In previous studies, it was sometimes defined as not having gone out for a certain period of time, such as a week or month $[19,20]$. In addition, the definition of HB is not only related to the concept of time, but also to the cause of $\mathrm{HB}$, such as the absence of assistance from others to go out or impairments of function to go out $[18,21]$. The definition of $\mathrm{HB}$ has changed over time. Thus, there are limitations in comparing the results between countries and studies. For example, in the United States, the HB prevalence rate among non-institutionalized Medicare beneficiaries aged 65 years and older using one question about the frequency of going out (completely or mostly HB) was 5.6\% [22]. Another U.S. study defined HB as self-reported ambulatory disability without any restriction of additional medical needs; $19.6 \%$ of older adults were HB [5]. In Japan, the prevalence rate of $\mathrm{HB}$ in 5000 older adults, randomly extracted using one item with frequency of going out being less than once a week, was 14.4\% [23]. In France, the prevalence of HB was $4.7 \%$, measured by asking persons if they are usually compelled to stay inside the home permanently (excluding an accident or temporary illness), but are not bedridden [9]. HB was divided into $\mathrm{HB}$ and non- $\mathrm{HB}$ and then further subdivided into $\mathrm{HB}$, semi- $\mathrm{HB}$, and non-HB $[20,22,24-26]$. As the classification criteria for semi-HB has varied in previous studies, the prevalence of semi-HB also varies [20,22,24-26]. Thus, an in-depth review of the definition and measurement of HB is needed prior to conducting studies to develop interventions to prevent HB.

Previous studies have investigated factors affecting HB in various ways, such as personal [19,23,27-30], social [2,31], and environmental factors [32]. Along with the changes in the definition of $\mathrm{HB}$, it is expected that the view of HB's influencing factors will also have changed; however, few studies have reviewed the factors affecting $\mathrm{HB}$ in older adults. $\mathrm{HB}$ can be influenced not only by individual factors but also by social relationships, social support systems, and environmental factors [33]. Therefore, it is necessary to review the factors affecting HB using an ecological approach [20]. Through this, we propose the implications of and an approach to interventions to prevent HB.

Scoping review is a new methodology for synthesizing the results of previous studies. It is excellent at identifying differences in knowledge, broadening knowledge, clarifying concepts, and investigating results [34]. It can provide an overview of a concept, and is useful in answering broad research questions [35]. Therefore, in this study, it was considered appropriate to conduct a scoping review to explore how the definition of $\mathrm{HB}$ has changed, how it is being measured, and the related factors that influence it.

\section{Materials and Methods}

\subsection{Design}

This scoping review utilized the five-stage methodological framework for scoping studies: (1) identifying the research question, (2) identifying relevant studies, (3) selecting studies, (4) charting the data, and (5) summarizing and reporting the findings [36]. We performed the study in accordance with these stages and performed Steps 4 and 5 simultaneously. 


\subsection{Identifying the Research Question}

This review aimed to answer three questions: (1) How is HB defined? This question aimed at determining how definitions differ by various components, such as the times or regions. (2) How is HB measured? Through this question, we tried to identify any differences in the measurement and methods of HB. (3) What are the factors that influence $\mathrm{HB}$ ? The last question was aimed at identifying the factors affecting $\mathrm{HB}$, and to classify them systematically. We did not define HB because we wanted to compare the definitions and measurements used in previous studies. In addition, to answer our research questions, we included a broad range of determinants and categorized them based on the revised ecological model [20].

\subsection{Identifying Relevant Studies}

We searched four databases, including a domestic (Korean) database and three international databases. The domestic database used was the Research Information Sharing Service, and international databases were PubMed, the Cumulative Index to Nursing and Allied Health Literature database, and Embase. A literature search was conducted for two weeks from 30 January 2020. We used a combination of search terms referred to in the PICO model. Regarding inclusion criteria, older people were selected for the study. Interventions and comparisons were not considered in this study. As our aim was to explore the definition, measurement, and factors affecting HB, we did not establish an intervention and comparison group to explore studies using various research. Finally, in order to explore the influencing factors of $\mathrm{HB}$, a study with $\mathrm{HB}$ as the outcome was considered in the inclusion criteria. The following search terms were used based on the MeSH (Medical Subject Headings) term: "aged" AND ("social isolation" OR "homebound person") AND "health" The reason the keyword was set to "aged" without limiting the target age to above 65 was because some countries defined those aged 50 or older as old adults. Data retrieval was conducted without limiting the publication date.

\subsection{Selecting Studies}

We selected the papers according to the following inclusion criteria: language, design, publication type, and outcome. We included both English and Korean studies and all research designs. We only included published articles, excluding editorials, book reviews, and poster presentations. In addition, we included a wide range of studies related to older people's HB. We selected papers for two-step screening by title, abstract, and full text.

The selection process is illustrated in Figure 1. A total of 14,158 articles were identified from the four databases. Of the total number of articles, 10,061 duplicate articles were removed. After screening the titles and abstracts, 4010 articles were excluded, leaving 87 articles. After checking for eligibility, 47 articles were finally included in the scoping review.

\subsection{Charting the Data and Summarizing and Reporting the Findings}

Each eligible study was charted and summarized using a standardized form, including author, publication year, design, participants, age, number of participants, country, definition, and theoretical model. In addition, the definitions of $\mathrm{HB}$ and the measurements used were analyzed. The measurements of $\mathrm{HB}$ were charted according to the measurement period, frequency, and meaning of "outdoor activities." The factors affecting HB were analyzed according to the framework presented in Table 1. The framework consisted of authors referring to the revised ecological model [20] and three factors: individual, social, and environmental. There were six categories of individual factors: demographic, health characteristics (illness), physical function, psychological function, cognitive function, and health behavior. 

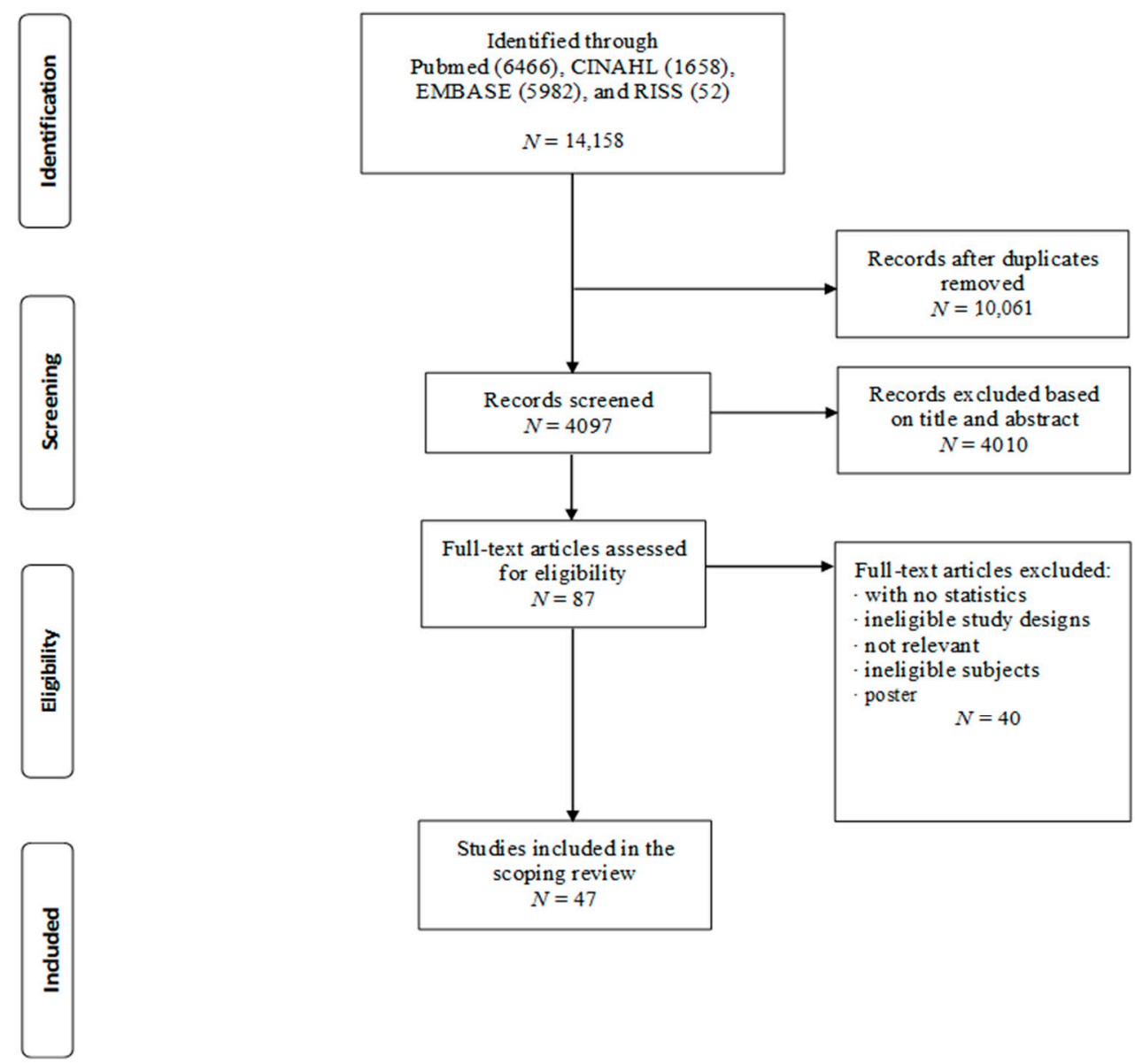

Figure 1. PRISMA flowchart of article selection.

Table 1. Analysis framework of determinants of HB.

\begin{tabular}{|c|c|c|}
\hline Factor & Category & Examples \\
\hline \multirow{6}{*}{ Individual factor } & Demographic & $\begin{array}{l}\text { Age, gender, race, marital status, education, } \\
\text { occupation, income, health insurance, etc. }\end{array}$ \\
\hline & Health characteristics & $\begin{array}{l}\text { Height, weight, body mass index, comorbidity, } \\
\text { illness, sarcopenia, laboratory analysis, etc. }\end{array}$ \\
\hline & Physical function & $\begin{array}{c}\text { Activities of daily living, Instrumental } \\
\text { activities of daily living, mobility difficulty, } \\
\text { hearing and vision ability, etc. }\end{array}$ \\
\hline & Psychological function & Mental health, depression, anxiety, phobia, etc. \\
\hline & Cognitive function & Cognitive impairment \\
\hline & Health behavior & Exercise, eating habits, smoking, drinking, etc. \\
\hline Social factor & & $\begin{array}{c}\text { Social participation, social support, social } \\
\text { activity, social capital, social isolation, contact } \\
\text { with various community health and } \\
\text { social services, etc. }\end{array}$ \\
\hline Environmental factor & & $\begin{array}{c}\text { Use of a mobility device to get around, stairs or } \\
\text { steps at the entrance, population size, } \\
\text { size of area, etc. }\end{array}$ \\
\hline
\end{tabular}




\section{Results}

\subsection{General Characteristics of the Study}

The general characteristics of previous studies regarding HB are shown in Table 2. Two-thirds of the 47 studies were cross-sectional studies, and $68.1 \%$ were older people. Regarding age, $78.7 \%$ of the participants were over 65 years, and the number of participants was below 1000 in $46.8 \%$ of the studies. Most of the studies were conducted in the United States and Asia, and 34.0\% have been conducted since 2010. Among the studies, $72.3 \%$ presented a definition of $\mathrm{HB}$, and $8.5 \%$ used a theoretical model.

Table 2. General characteristics of previous studies regarding HB $(N=47)$.

\begin{tabular}{|c|c|c|c|}
\hline Characteristics & Category & $N$ & $\%$ \\
\hline \multirow{2}{*}{ Design } & Longitudinal study & 16 & 34.0 \\
\hline & Cross-sectional study & 31 & 66.0 \\
\hline \multirow{2}{*}{ Participants } & Older people & 32 & 68.1 \\
\hline & HB older people & 15 & 31.9 \\
\hline \multirow{2}{*}{ Age } & Above 50-64 & 10 & 21.3 \\
\hline & Above 65 & 37 & 78.7 \\
\hline \multirow{3}{*}{ Number of participants } & $\leq 1000$ & 22 & 46.8 \\
\hline & $1000 \sim 2000$ & 8 & 17.0 \\
\hline & $\geq 2000$ & 17 & 36.2 \\
\hline \multirow{3}{*}{ Country } & Asia & 18 & 38.3 \\
\hline & Europe & 9 & 19.1 \\
\hline & America & 20 & 42.6 \\
\hline \multirow{2}{*}{ Publication year } & 2010-present & 16 & 34.0 \\
\hline & $2001-2009$ & 31 & 66.0 \\
\hline Definition & Yes & 34 & 72.3 \\
\hline Theoretical model & Yes & 4 & 8.5 \\
\hline
\end{tabular}

\subsection{Definition of HB and the Applied Conceptual Model}

Table 3 shows studies that present the definition of HB and the applied conceptual framework of HB. Most researchers defined HB as "a condition characterized by an infrequency of going outdoors" $[6,9,19-22,26,32,37-39]$. Other researchers defined HB as being "confined to one's home" [17,18], "remaining inside or just around the home [during] daily life" [40], or "being compelled to stay inside one's home permanently excluding an accident or temporary illness" [9].

In some studies, the cause or situation of $\mathrm{HB}$ was specified in the definition of HB [18]. The boundary of the daily life of older adults is limited to home because of immobility [18,21,40], medical issues [21], or either help or taxing effort is needed to leave the house [21,40]. Koyama and colleagues defined the reason for being HB as not only physical but also psychological or geographical [6]. Chinese researchers [39] viewed HB as a continuous spectrum (line) of living activities. They included, in the concept of HB, not only being physically confined to the house, but also the degree of help needed to leave the house and whether an individual had social contact.

The application of a conceptual framework to explain HB began in 2001. HB is closely related to the aging process [40]; thus, HB refers to a condition in which physical and social independence are impaired, and dependence on others increases due to chronic physical illness and disability. Ornstein et al. applied a gerontological conceptual framework to explain HB. They explained that the impact of disability is based on the confluence of personal capacity and the ability of social support to compensate for capacity limitations [22]. 
Xiang et al. explained HB using the International Classification of Functioning, Disability, and Health (ICF) and the concept of autonomy [32]. They defined HB as a result of interactions between body functions and structures, activities, participation, and environmental factors [6,41]. In other words, $\mathrm{HB}$ is defined as one of the states of a continuum of living activities. Xiang et al. applied a revised ecological model of aging [32] to determine the influencing factors that cause HB [20]. These include demographic (age, gender, race, and ethnicity), socioeconomic (such as education and income), social (such as living arrangements, social networks, and social support), and psychosocial factors (e.g., self-efficacy), health behaviors, health conditions, functioning (physical and cognitive), and environment (built and physical). This is a point of view that considers not only individual factors but also social and environmental factors as the reasons for $\mathrm{HB}$.

Table 3. Definition of HB and the applied theoretical model.

\begin{tabular}{|c|c|c|c|c|c|c|c|}
\hline \multirow[b]{2}{*}{ Author } & \multirow[b]{2}{*}{ Year } & \multirow[b]{2}{*}{ Country } & \multirow[b]{2}{*}{$\begin{array}{c}\text { Definition } \\
\text { (Conceptual Framework) }\end{array}$} & \multirow[b]{2}{*}{ Operational Definition } & \multicolumn{3}{|c|}{ Going Out of the House } \\
\hline & & & & & $\begin{array}{l}\text { Observation } \\
\text { Period for } \\
\text { Measure }\end{array}$ & Frequency & $\begin{array}{l}\text { Meaning of } \\
\text { "Outdoor } \\
\text { Activities" }\end{array}$ \\
\hline $\begin{array}{c}\text { Bruce \& } \\
\text { McNamara [17] }\end{array}$ & 1992 & US & Being confined to the home & $\begin{array}{l}\text { Two items: individual } \\
\text { stayed in their bed or in a } \\
\text { chair for most or all of the } \\
\text { day during the last two } \\
\text { weeks or they stayed } \\
\text { indoors for most or all of } \\
\text { the day during the last } \\
\text { two weeks. }\end{array}$ & 2 weeks & & \\
\hline $\begin{array}{l}\text { Lindesay \& } \\
\text { Thomson [18] }\end{array}$ & 1993 & UK & $\begin{array}{l}\text { Being neither completely } \\
\text { housebound nor } \\
\text { blockbound or only going } \\
\text { out of doors with the } \\
\text { assistance of others }\end{array}$ & $\begin{array}{l}\text { Individuals were } \\
\text { considered housebound if } \\
\text { they met the following two } \\
\text { criteria: (1) they were } \\
\text { either completely } \\
\text { housebound or } \\
\text { blockbound (for at least } \\
\text { one month) and (2) } \\
\text { interviewers judged that } \\
\text { their current housebound } \\
\text { or blockbound status was } \\
\text { likely to be permanent. }\end{array}$ & 1 month & Never & $\begin{array}{l}\text { Individual goes } \\
\text { beyond their } \\
\text { door or the } \\
\text { boundary of } \\
\text { the block }\end{array}$ \\
\hline Ganduli et al. [19] & 1996 & US & $\begin{array}{c}\text { Leaving the house once } \\
\text { per week or less }\end{array}$ & $\begin{array}{l}\text { One item: "How often do } \\
\text { you get out of the house?" }\end{array}$ & & $\begin{array}{c}\text { Once per } \\
\text { week or less }\end{array}$ & \\
\hline Engberg et al. [21] & 2001 & US & $\begin{array}{l}\text { Being confined to the home } \\
\text { Homebound (HB)older } \\
\text { adults are those who, due } \\
\text { to medical conditions } \\
\text { and/or mobility-affecting } \\
\text { impairments, are not able } \\
\text { to freely leave their homes, } \\
\text { and require help in } \\
\text { doing so }\end{array}$ & $\begin{array}{l}\text { Health Care Financing } \\
\text { Agency Criteria }\end{array}$ & & & \\
\hline $\begin{array}{c}\text { Kono \& } \\
\text { Kanagawa [37] }\end{array}$ & 2001 & Japan & $\begin{array}{l}\text { Being HB was defined } \\
\text { according to the frequency } \\
\text { of getting out and mobility. }\end{array}$ & $\begin{array}{l}\text { Self-reported behaviors } \\
\text { (10 behaviors) regarding } \\
\text { getting out within a week }\end{array}$ & 1 week & $\begin{array}{l}\text { Never/once } \\
\text { per week }\end{array}$ & $\begin{array}{l}\text { Ten behaviors: } \\
\text { go to adult day } \\
\text { care, going out } \\
\text { to the garden, } \\
\text { taking a walk, } \\
\text { and so on. }\end{array}$ \\
\hline Inoue et al. [40] & 2001 & Japan & $\begin{array}{l}\text { The state of remaining } \\
\text { inside or just around the } \\
\text { home during daily life } \\
\text { (Aging process: the } \\
\text { development of a chronic } \\
\text { physical illness and } \\
\text { disability in the elderly } \\
\text { often results in a loss of } \\
\text { physical and social } \\
\text { independence and } \\
\text { increased dependence } \\
\text { on others) }\end{array}$ & $\begin{array}{l}\text { One item: "In your daily } \\
\text { life, do you leave home } \\
\text { without assistance } \\
\text { from others?" }\end{array}$ & & Never & \\
\hline Sharkey et al. [42] & 2002 & US & $\begin{array}{l}\text { Medicare classified a } \\
\text { person as HB if leaving the } \\
\text { home requires } \\
\text { considerable effort and } \\
\text { occurs infrequently due to } \\
\text { an illness or injury. }\end{array}$ & & & & \\
\hline
\end{tabular}


Table 3. Cont.

\begin{tabular}{|c|c|c|c|c|c|c|c|}
\hline \multirow[b]{2}{*}{ Author } & \multirow[b]{2}{*}{ Year } & \multirow[b]{2}{*}{ Country } & \multirow[b]{2}{*}{$\begin{array}{c}\text { Definition } \\
\text { (Conceptual Framework) }\end{array}$} & \multirow[b]{2}{*}{ Operational Definition } & \multicolumn{3}{|c|}{ Going Out of the House } \\
\hline & & & & & $\begin{array}{l}\text { Observation } \\
\text { Period for } \\
\text { Measure }\end{array}$ & Frequency & $\begin{array}{l}\text { Meaning of } \\
\text { "Outdoor } \\
\text { Activities" }\end{array}$ \\
\hline $\begin{array}{l}\text { Kawamura } \\
\text { et al. [37] }\end{array}$ & 2005 & Japan & $\begin{array}{c}\text { A condition characterized } \\
\text { by an infrequency of going } \\
\text { outdoors } \\
\text { Four categories of HB: by } \\
\text { social contract with friends, } \\
\text { neighbors, or relatives } \\
\text { other than live-in family } \\
\text { members and } \\
\text { with/without assistance }\end{array}$ & $\begin{array}{l}\text { One item: frequency of } \\
\text { leaving the house (1) once } \\
\text { per day or more, (2) once } \\
\text { every 2-3 days, (3) about } \\
\text { once per week, (4) rarely. }\end{array}$ & & $\begin{array}{l}\text { once per } \\
\text { week } \\
\text { or rarely }\end{array}$ & $\begin{array}{l}\text { Leaving the } \\
\text { house including } \\
\text { going to the } \\
\text { surrounding } \\
\text { gardens } \\
\text { or grounds }\end{array}$ \\
\hline $\begin{array}{c}\text { Choi \& } \\
\text { McDougal [43] }\end{array}$ & 2007 & US & $\begin{array}{l}\text { HB older adults are those } \\
\text { who, due to medical } \\
\text { conditions and/or } \\
\text { mobility-affecting } \\
\text { impairments, are not able } \\
\text { to freely leave their homes } \\
\text { and require help in } \\
\text { doing so }\end{array}$ & & & & \\
\hline $\begin{array}{l}\text { Katsumata } \\
\text { et al. [44] }\end{array}$ & 2007 & Japan & & $\begin{array}{l}\text { One item: frequency of } \\
\text { going outdoors }\end{array}$ & & $\begin{array}{c}\text { Once per } \\
\text { week or less }\end{array}$ & \\
\hline Locher et al. [45] & 2008 & US & $\begin{array}{l}\text { Medicare definition of HB } \\
\text { status: [An] individual } \\
\text { [who] has a condition ... } \\
\text { that restricts [their] ability } \\
\text { to leave home except with } \\
\text { the assistance of another } \\
\text { individual or the aid of a } \\
\text { supportive device or [who] } \\
\text { has a condition [where] } \\
\text { leaving home is medically } \\
\text { contraindicated }\end{array}$ & & & & \\
\hline $\begin{array}{l}\text { Cohen- } \\
\text { Mansfield [8] }\end{array}$ & 2010 & Israel & & $\begin{array}{l}\text { One item: how often do } \\
\text { they go outside of their } \\
\text { home (more than once per } \\
\text { week, or once a week } \\
\text { or less) }\end{array}$ & & $\begin{array}{l}\text { Once per } \\
\text { week or less }\end{array}$ & \\
\hline $\begin{array}{l}\text { Murayama } \\
\text { et al. [29] }\end{array}$ & 2012 & Japan & & $\begin{array}{l}\text { One item: “How often do } \\
\text { you usually go outside the } \\
\text { house?" (once a week or } \\
\text { less vs. more than once } \\
\text { per week) }\end{array}$ & Usually & $\begin{array}{l}\text { Once per } \\
\text { week or less }\end{array}$ & \\
\hline $\begin{array}{c}\text { Cohen- } \\
\text { Mansfield [46] }\end{array}$ & 2012 & Israel & & $\begin{array}{l}\text { One item: "How often do } \\
\text { you go outside of your } \\
\text { home?" (more than once } \\
\text { per week, or once a week } \\
\text { or less) }\end{array}$ & & $\begin{array}{l}\text { Once per } \\
\text { week or less }\end{array}$ & \\
\hline Choi et al. [28] & 2012 & $\begin{array}{l}\text { South } \\
\text { Korea }\end{array}$ & & $\begin{array}{l}\text { One item: "How often do } \\
\text { you go outside of your } \\
\text { home?" (more than once } \\
\text { per week, or once a week } \\
\text { or less) }\end{array}$ & 1 week & $\begin{array}{l}\text { Never } \\
\text { within } \\
\text { a week }\end{array}$ & $\begin{array}{l}\text { Go shopping or } \\
\text { walking, visit } \\
\text { the hospital or } \\
\text { center (excludes } \\
\text { leaving the } \\
\text { house for a } \\
\text { short period, for } \\
\text { instance, to take } \\
\text { out the trash) }\end{array}$ \\
\hline Herr et al. [9] & 2013 & France & $\begin{array}{l}\text { Remained inside their } \\
\text { homes during the previous } \\
\text { week or if they went out at } \\
\text { all, only for health } \\
\text { care purposes }\end{array}$ & $\begin{array}{l}\text { One item for the } \\
\text { non-bedridden: “Are you } \\
\text { usually compelled to stay } \\
\text { inside your home } \\
\text { permanently (excluding an } \\
\text { accident or } \\
\text { temporary illness)?” }\end{array}$ & Usually & $\begin{array}{l}\text { Never } \\
\text { within } \\
\text { a week }\end{array}$ & $\begin{array}{l}\text { Accident or tem- } \\
\text { porary illness }\end{array}$ \\
\hline $\begin{array}{l}\text { Umegaki } \\
\text { et al. [23] }\end{array}$ & 2015 & Japan & & $\begin{array}{l}\text { One item: frequency of } \\
\text { excursions within a week }\end{array}$ & & $\begin{array}{l}\text { Less than } \\
\text { once } \\
\text { per week }\end{array}$ & \\
\hline
\end{tabular}


Table 3. Cont.

\begin{tabular}{|c|c|c|c|c|c|c|c|}
\hline \multirow[b]{2}{*}{ Author } & \multirow[b]{2}{*}{ Year } & \multirow[b]{2}{*}{ Country } & \multirow[b]{2}{*}{$\begin{array}{c}\text { Definition } \\
\text { (Conceptual Framework) }\end{array}$} & \multirow[b]{2}{*}{ Operational Definition } & \multicolumn{3}{|c|}{ Going Out of the House } \\
\hline & & & & & $\begin{array}{l}\text { Observation } \\
\text { Period for } \\
\text { Measure }\end{array}$ & Frequency & $\begin{array}{l}\text { Meaning of } \\
\text { "Outdoor } \\
\text { Activities" }\end{array}$ \\
\hline Musich et al. [5] & 2015 & US & $\begin{array}{l}\text { Ambulatory disability } \\
\text { without the restriction of } \\
\text { additional medical needs }\end{array}$ & $\begin{array}{l}\text { Self-reported ambulatory } \\
\text { disability (5 items): HB } \\
\text { state was identified by } \\
\text { answering "yes" to any of } \\
\text { the following five items: (1) } \\
\text { have trouble getting } \\
\text { around at home or outside } \\
\text { your home; (2) use a cane, } \\
\text { wheelchair or walker to } \\
\text { move around at home or } \\
\text { outside your home; (3) } \\
\text { need help from another } \\
\text { person to move around } \\
\text { inside or outside your } \\
\text { home; (4) need to stay in } \\
\text { the house most or all of the } \\
\text { time; (5) need to stay in } \\
\text { bed most or all of the time }\end{array}$ & & $\begin{array}{l}\text { Any } \\
\text { ambulatory } \\
\text { disability }\end{array}$ & \\
\hline Ornstein et al. [22] & 2015 & US & $\begin{array}{l}\text { HB: never or rarely left } \\
\text { home; semi-HB: only left } \\
\text { home with assistance or } \\
\text { had difficulty or needed } \\
\text { helping to leave home } \\
\text { (Gerontological conceptual } \\
\text { framework: late-life } \\
\text { disability - the impact of } \\
\text { disability is based on the } \\
\text { confluence of personal } \\
\text { capacity and the ability of } \\
\text { social support to } \\
\text { compensate for } \\
\text { capacity limitations) }\end{array}$ & $\begin{array}{l}\text { Three items: (1) how often } \\
\text { they left home to go } \\
\text { outside in the last month } \\
\text { (daily, most days (5-6 days } \\
\text { per week), some days (2-4 } \\
\text { days per week), rarely } \\
\text { (once per week or less), or } \\
\text { never); (2) they were asked } \\
\text { whether they needed } \\
\text { assistance; (3) they were } \\
\text { asked if they were ever } \\
\text { able to go out by } \\
\text { themselves, or they } \\
\text { reported going outside } \\
\text { without help then reported } \\
\text { whether they had difficulty } \\
\text { doing the activity alone } \\
\text { (regardless of the use of } \\
\text { assistive devices) in the } \\
\text { last month. }\end{array}$ & Previous month & $\begin{array}{l}\text { Never or } \\
\text { rarely } \\
(\leq 1 \text { day }) \\
\text { within } \\
\text { a week }\end{array}$ & \\
\hline Takhashi et al. [31] & 2015 & Japan & $\begin{array}{l}\text { A condition characterized } \\
\text { by an infrequency of } \\
\text { going outdoors }\end{array}$ & $\begin{array}{l}\text { One item: frequency of } \\
\text { going outdoors }\end{array}$ & & $\begin{array}{l}\text { Once per } \\
\text { week or less }\end{array}$ & \\
\hline Koyama et al. [47] & 2016 & Japan & $\begin{array}{l}\text { Leaving home less often } \\
\text { than once weekly, which } \\
\text { reflects not only physical } \\
\text { reasons for being confined } \\
\text { to one's home, but also } \\
\text { psychological or } \\
\text { geographical reasons }\end{array}$ & $\begin{array}{l}\text { One item pertaining to } \\
\text { frequency of going } \\
\text { outdoors: "How often do } \\
\text { you usually go outside } \\
\text { the house?" }\end{array}$ & Usually & $\begin{array}{l}\text { Less than } \\
\text { once } \\
\text { per week }\end{array}$ & $\begin{array}{c}\text { Shopping, } \\
\text { meeting up } \\
\text { with people, } \\
\text { walking, } \\
\text { visiting the } \\
\text { hospital, and } \\
\text { other activities }\end{array}$ \\
\hline $\begin{array}{l}\text { Negron-Blanco } \\
\text { et al. [30] }\end{array}$ & 2016 & Spain & $\begin{array}{l}\text { Having severe or extreme } \\
\text { difficulty getting out of } \\
\text { the house }\end{array}$ & $\begin{array}{c}\text { One item from } \\
\text { WHODAS-36: "In the past } \\
30 \text { days, how much } \\
\text { difficulty did you have } \\
\text { leaving home?" } \\
\text { None/mild/moderate/ } \\
\text { severe/extreme or cannot } \\
\text { do, with a response of } \\
\text { "severe" or "extreme or } \\
\text { cannot do" was construed } \\
\text { as being HB. }\end{array}$ & Past 30 days & $\begin{array}{l}\text { Severe or } \\
\text { extreme/ } \\
\text { cannot } \\
\text { leave home }\end{array}$ & \\
\hline $\begin{array}{l}\text { Hamazake } \\
\text { et al. [10] }\end{array}$ & 2016 & Japan & & $\begin{array}{l}\text { One item: "Do you go out } \\
\text { more than once per week?" }\end{array}$ & & $\begin{array}{l}\text { Less than } \\
\text { once } \\
\text { per week }\end{array}$ & \\
\hline Harada et al. [48] & 2016 & Japan & $\begin{array}{l}\text { Going outdoors less than } \\
\text { once per week }\end{array}$ & $\begin{array}{l}\text { One item: "Do you usually } \\
\text { go outside the house at } \\
\text { least once per week?" }\end{array}$ & Usually & $\begin{array}{l}\text { Once } \\
\text { a week }\end{array}$ & \\
\hline De-Rosende [49] & 2017 & Spain & $\begin{array}{l}\text { Considered HB state if the } \\
\text { individual remained inside } \\
\text { their home during the } \\
\text { previous week or if they } \\
\text { went out only for health } \\
\text { care purposes (e.g., } \\
\text { medical consultation or } \\
\text { health emergencies) }\end{array}$ & $\begin{array}{l}\text { One item: the number of } \\
\text { days on which they left } \\
\text { home during the } \\
\text { previous week }\end{array}$ & Previous week & $\begin{array}{l}\text { Never or } \\
\text { only for } \\
\text { health care } \\
\text { purposes }\end{array}$ & \\
\hline
\end{tabular}


Table 3. Cont

\begin{tabular}{|c|c|c|c|c|c|c|c|}
\hline \multirow[b]{2}{*}{ Author } & \multirow[b]{2}{*}{ Year } & \multirow[b]{2}{*}{ Country } & \multirow[b]{2}{*}{$\begin{array}{c}\text { Definition } \\
\text { (Conceptual Framework) }\end{array}$} & \multirow[b]{2}{*}{ Operational Definition } & \multicolumn{3}{|c|}{ Going Out of the House } \\
\hline & & & & & $\begin{array}{c}\text { Observation } \\
\text { Period for } \\
\text { Measure }\end{array}$ & Frequency & $\begin{array}{l}\text { Meaning of } \\
\text { "Outdoor" } \\
\text { Activities" }\end{array}$ \\
\hline Soones et al. [26] & 2017 & US & $\begin{array}{l}\text { HB (never or rarely left } \\
\text { home in the last month), } \\
\text { semi-HB (only left home } \\
\text { with assistance; needed } \\
\text { help or had difficulty), } \\
\text { non-HB (left home without } \\
\text { help or difficulty) } \\
\text { (Aday and Andersen's } \\
\text { Behavioral Model of } \\
\text { Health Service Use and } \\
\text { gerontological frameworks } \\
\text { for the study of HB state } \\
\text { due to disability) }\end{array}$ & $\begin{array}{l}\text { Three items: "How often } \\
\text { did you go out in the last } \\
\text { month?" "Did anyone ever } \\
\text { help you?" and "How } \\
\text { much difficulty did you } \\
\text { have leaving the house } \\
\text { by yourself?" }\end{array}$ & Previous month & $\begin{array}{l}\text { Never } \\
\text { or rarely }\end{array}$ & \\
\hline Jing et al. [27] & 2017 & China & $\begin{array}{l}\text { People who leave home } \\
\text { less than once per week }\end{array}$ & $\begin{array}{l}\text { Record of occasions on } \\
\text { which the individual went } \\
\text { out during the month } \\
\text { before the survey (In cases } \\
\text { where the going out counts } \\
\text { for each week of the month } \\
\text { differed, the total count for } \\
\text { that month averaged by } \\
\text { the number of weeks was } \\
\text { considered) }\end{array}$ & Previous month & $\begin{array}{l}\text { Less than } \\
\text { once } \\
\text { per week }\end{array}$ & \\
\hline $\begin{array}{c}\text { Xiang \& } \\
\text { Brooks [25] }\end{array}$ & 2017 & US & $\begin{array}{l}\text { "Never" or "rarely" left } \\
\text { home in the last month } \\
\text { HB, semi-HB: 1) they } \\
\text { received help leaving } \\
\text { home and would "never," } \\
\text { "rarely," or "sometimes" go } \\
\text { outside by themselves or 2) } \\
\text { they did not receive help } \\
\text { leaving home but reported } \\
\text { "a lot," "some," or "a little" } \\
\text { difficulty leaving home } \\
\text { by themselves) }\end{array}$ & $\begin{array}{l}\text { Three items: (1) how often } \\
\text { they left home to go } \\
\text { outside in the last month } \\
\text { [daily, most days (5-6 days } \\
\text { per week), some days (2-4 } \\
\text { days per week), rarely } \\
\text { (once per week or less), or } \\
\text { never]; (2) they were asked } \\
\text { whether they needed } \\
\text { assistance; (3) they were } \\
\text { asked if they were ever } \\
\text { able to go out by } \\
\text { themselves, or they } \\
\text { reported going outside } \\
\text { without help then reported } \\
\text { whether they had difficulty } \\
\text { doing the activity alone } \\
\text { (regardless of the use of } \\
\text { assistive devices) in the } \\
\text { last month. }\end{array}$ & Previous month & $\begin{array}{l}\text { Never or } \\
\text { rarely } \\
(\leq 1 \text { day }) \\
\text { within } \\
\text { a week }\end{array}$ & \\
\hline Uemura et al. [2] & 2018 & Japan & $\begin{array}{l}\text { Going outdoors less than } \\
\text { once per week }\end{array}$ & $\begin{array}{l}\text { One item: "Do you go out } \\
\text { at least once per week?" }\end{array}$ & & $\begin{array}{l}\text { At least } \\
\text { once } \\
\text { per week }\end{array}$ & \\
\hline Meng et al. [39] & 2018 & China & $\begin{array}{l}\text { Going out of the house } \\
\text { once per week or less }\end{array}$ & $\begin{array}{l}\text { Four items: (1) “Do you } \\
\text { spend more time at home } \\
\text { than going out, and is this } \\
\text { the norm?" (2) “How } \\
\text { many times do you go out } \\
\text { to shop, walk, or visit the } \\
\text { hospital?" [(1) More than } \\
\text { once per day, (2) 2-3 days } \\
\text { at a time, (3) once per } \\
\text { week, (4) hardly ever go } \\
\text { out] (3) “How often do you } \\
\text { meet or communicate with } \\
\text { friends, neighbors, or } \\
\text { relatives outside the } \\
\text { home?" [(1) 2-3 days at a } \\
\text { time (2) once per week (3) } \\
\text { once per month (4) hardly } \\
\text { ever], (4) “If you go out, do } \\
\text { you need help?" } \\
\text { Higher scores indicate } \\
\text { severity of the HB status. }\end{array}$ & & & \\
\hline
\end{tabular}


Table 3. Cont.

\begin{tabular}{|c|c|c|c|c|c|c|c|}
\hline \multirow[b]{2}{*}{ Author } & \multirow[b]{2}{*}{ Year } & \multirow[b]{2}{*}{ Country } & \multirow[b]{2}{*}{$\begin{array}{c}\text { Definition } \\
\text { (Conceptual Framework) }\end{array}$} & \multirow[b]{2}{*}{ Operational Definition } & \multicolumn{3}{|c|}{ Going Out of the House } \\
\hline & & & & & $\begin{array}{l}\text { Observation } \\
\text { Period for } \\
\text { Measure }\end{array}$ & Frequency & $\begin{array}{l}\text { Meaning of } \\
\text { "Outdoor } \\
\text { Activities" }\end{array}$ \\
\hline Sakurai et al. [6] & 2019 & Japan & & $\begin{array}{l}\text { One item: "How often do } \\
\text { you usually go outdoors?" } \\
\text { (Twice daily or more, daily, } \\
\text { about once every } 2-3 \text { days, } \\
\text { about once per week or } \\
\text { less often) }\end{array}$ & Usually & $\begin{array}{l}\text { Every few } \\
\text { days or less } \\
\text { within } \\
\text { a week }\end{array}$ & $\begin{array}{c}\text { Going } \\
\text { shopping, } \\
\text { talking a walk, } \\
\text { visiting the } \\
\text { hospital, or } \\
\text { going out to } \\
\text { work or to } \\
\text { participate in } \\
\text { social activities }\end{array}$ \\
\hline Zhao et al. [24] & 2019 & & $\begin{array}{l}\text { Participants were defined } \\
\text { as totally HB if they never } \\
\text { or rarely left home. } \\
\text { Semi-HB participants were } \\
\text { those who needed help } \\
\text { leaving home and would } \\
\text { "never," "rarely," or } \\
\text { "sometimes" go out by } \\
\text { themselves, or they had "a } \\
\text { lot," "some," or "a little" } \\
\text { difficulty going out by } \\
\text { themselves without help. }\end{array}$ & $\begin{array}{l}\text { Three items: (1) how often } \\
\text { they left home to go } \\
\text { outside in the last month } \\
\text { [daily, most days (5-6 days } \\
\text { per week), some days (2-4 } \\
\text { days per week), rarely } \\
\text { (once a week or less), or } \\
\text { never]; (2) whether they } \\
\text { needed assistance; (3) if } \\
\text { they were ever able to go } \\
\text { out by themselves, or they } \\
\text { reported going outside } \\
\text { without help then reported } \\
\text { whether they had difficulty } \\
\text { doing the activity alone } \\
\text { (regardless of the use of } \\
\text { assistive devices) in the } \\
\text { last month. }\end{array}$ & Previous month & $\begin{array}{l}\text { Never or } \\
\text { rarely } \\
(\leq 1 \text { day }) \\
\text { within } \\
\text { a week }\end{array}$ & \\
\hline Xiang et al. [32] & 2020 & US & $\begin{array}{l}\text { Never or rarely leave the } \\
\text { house. HB status defined } \\
\text { on a continuum of outdoor } \\
\text { mobility determined by } \\
\text { physical capacity, } \\
\text { availability of social } \\
\text { support, and degree of } \\
\text { autonomy. } \\
\text { Determined the } \\
\text { classification of HB state } \\
\text { (HB, semi-HB, or } \\
\text { non-HBd) by the frequency } \\
\text { of going out, physical } \\
\text { capacity, availability of } \\
\text { social support, and degree } \\
\text { of autonomy. } \\
\text { (International } \\
\text { Classification of } \\
\text { Functioning, Disability } \\
\text { and Health and the } \\
\text { concept of autonomy) }\end{array}$ & $\begin{array}{l}\text { Four items: (1) "How often } \\
\text { did you go out in the last } \\
\text { month?" (responses on a } \\
\text { 5-point Likert scale: never, } \\
\text { rarely, some days, most } \\
\text { days, every day) (2) "Did } \\
\text { you ever have to stay in } \\
\text { because no one was there } \\
\text { to help you?" (3) "Did } \\
\text { anyone ever help you?" (4) } \\
\text { "How often did you go } \\
\text { outside by yourself?" }\end{array}$ & Previous month & $\begin{array}{l}\text { Never or } \\
\text { rarely } \\
(\leq 1 \text { day }) \\
\text { within } \\
\text { a week }\end{array}$ & \\
\hline Xiang et al. [20] & 2020 & US & $\begin{array}{l}\text { Never or rarely went out of } \\
\text { the home in the last month } \\
\text { (An ecological model } \\
\text { revised by Satariano [50]) }\end{array}$ & $\begin{array}{l}\text { One item: "How often do } \\
\text { you go outside of } \\
\text { your home?" }\end{array}$ & Previous month & $\begin{array}{l}\text { Never or } \\
\text { rarely } \\
(\leq 1 \text { day })\end{array}$ & \\
\hline
\end{tabular}

\subsection{Operational Definition of $H B$}

Most epidemiological researchers have investigated $\mathrm{HB}$ using self-reported questions about the frequency of going out for a certain period. The observation period for measuring the frequency of going out varies from one week $[28,37,50]$ to one month $[18,20,26,27,30]$ or usually $[9,47]$. The criteria for $\mathrm{HB}$ also varies according to the study, from not going out at all in some studies $[9,18,28,40,50]$ to going out less than once $[2,8,10,19,20,22,23,29,37,38,44,47]$. When defining $\mathrm{HB}$, the scope of the house also varies, from staying inside the building or staying on the block where the house is located (blockbound) [18] to staying in the house, including the garden or yard [38]. The meaning of "going out" varies as well. The activity of leaving the house to take out the trash is not considered going out, but activities that involve leaving home for a certain purpose, that is, going shopping, walking for leisure, visiting the hospital or center, commuting, and going to work or participating in social 
activities, were defined as going out [28,39]. In some studies, emergency visits due to accidents or temporary illnesses were not included in "going out" [9].

Researchers $[20,22,26]$ in the United States have classified HB into three groups: HB, semi-HB, and non-HB. HB was defined as never or rarely leaving home. Semi-HB refers to cases where individuals get or need help to go out, or it is difficult for them to go out by themselves. Non-HB is a case in which a person can go out without help. Meng et al. defined $\mathrm{HB}$ as going out less than once per day and calculated the severity of HB by adding up the amount of time a person spent at home; the frequency at which they went out, met, and communicated with friends, neighbors, and relatives outside their home; and how often they needed help when going out [39].

\subsection{Factors Affecting $H B$}

For the analysis of determinants, we categorized three factors based on the revised ecological model: individual, social, and environmental factors (Table 4). The individual factors were divided into six categories: demographic, health characteristics, physical function, psychological function, cognitive function, and health behavior. The effects of personal factors were investigated in all studies. Of the 16 studies, $10(62.5 \%)$ were identified as exploring the influence of social factors, and $6(37.5 \%)$ as exploring the influence of environmental factors.

Table 4. Determinants of HB state based on individual, social, and environmental factors.

\begin{tabular}{|c|c|c|c|c|c|c|c|c|c|c|}
\hline \multirow[b]{2}{*}{ Author } & \multirow[b]{2}{*}{ Year } & \multicolumn{6}{|c|}{ Individual } & \multirow[b]{2}{*}{ Social } & \multirow[b]{2}{*}{ Environmental } & \multirow[b]{2}{*}{ Framework } \\
\hline & & Demographic & $\begin{array}{c}\text { Health } \\
\text { Characteristic }\end{array}$ & $\begin{array}{l}\text { Physical } \\
\text { Function }\end{array}$ & $\begin{array}{l}\text { Psychological } \\
\text { Function }\end{array}$ & $\begin{array}{l}\text { Cognitive } \\
\text { Function }\end{array}$ & $\begin{array}{c}\text { Health } \\
\text { Behavior }\end{array}$ & & & \\
\hline Lindesay et al. [18]. & 1993 & $\mathrm{O}$ & $\mathrm{O}$ & $\mathrm{O}$ & $\mathrm{O}$ & & & $\mathrm{O}$ & $\mathrm{O}$ & \\
\hline Ganguli et al. [19] & 1996 & $\mathrm{O}$ & $\mathrm{O}$ & $\mathrm{O}$ & $\mathrm{O}$ & $\mathrm{O}$ & & $\mathrm{O}$ & & \\
\hline Inoue et al. [40] & 2001 & $\mathrm{O}$ & $\mathrm{O}$ & $\mathrm{O}$ & & & & & & \\
\hline Jensen et al. [51] & 2006 & $\mathrm{O}$ & $\mathrm{O}$ & $\mathrm{O}$ & $\mathrm{O}$ & & $\mathrm{O}$ & & & \\
\hline Katsumata et al. [44] & 2007 & $\mathrm{O}$ & $\mathrm{O}$ & $\mathrm{O}$ & & & & $\mathrm{O}$ & & \\
\hline Choi et al. [28] & 2012 & $\mathrm{O}$ & $\mathrm{O}$ & $\mathrm{O}$ & $\mathrm{O}$ & & & & & \\
\hline $\begin{array}{l}\text { Cohen-Mansfield } \\
\text { et al. [46] }\end{array}$ & 2012 & $\mathrm{O}$ & $\mathrm{O}$ & $\mathrm{O}$ & $\mathrm{O}$ & $\mathrm{O}$ & & & $\mathrm{O}$ & \\
\hline Murayama et al. [29] & 2012 & $\mathrm{O}$ & $\mathrm{O}$ & $\mathrm{O}$ & $\mathrm{O}$ & & $\mathrm{O}$ & $\mathrm{O}$ & $\mathrm{O}$ & \\
\hline Takahashi et al. [31] & 2015 & $\mathrm{O}$ & $\mathrm{O}$ & $\mathrm{O}$ & $\mathrm{O}$ & & & $\mathrm{O}$ & & \\
\hline Umegaki et al. [23] & 2015 & $\mathrm{O}$ & & & $\mathrm{O}$ & & & $\mathrm{O}$ & & \\
\hline Koyama et al. [47] & 2016 & $\mathrm{O}$ & $\mathrm{O}$ & $\mathrm{O}$ & $\mathrm{O}$ & & & & $\mathrm{O}$ & \\
\hline $\begin{array}{l}\text { Negron-Blanco } \\
\text { et al. [30] }\end{array}$ & 2016 & $\mathrm{O}$ & $\mathrm{O}$ & $\mathrm{O}$ & $\mathrm{O}$ & $\mathrm{O}$ & $\mathrm{O}$ & $\mathrm{O}$ & & \\
\hline $\begin{array}{l}\text { De-Rosende } \\
\text { et al. [49] }\end{array}$ & 2017 & $\mathrm{O}$ & $\mathrm{O}$ & $\mathrm{O}$ & $\mathrm{O}$ & & & & $\mathrm{O}$ & \\
\hline Jing et al. [27] & 2017 & $\mathrm{O}$ & $\mathrm{O}$ & $\mathrm{O}$ & $\mathrm{O}$ & & $\mathrm{O}$ & $\mathrm{O}$ & & \\
\hline Uemura et al. [2] & 2018 & $\mathrm{O}$ & $\mathrm{O}$ & $\mathrm{O}$ & $\mathrm{O}$ & & & $\mathrm{O}$ & & \\
\hline Xiang et al. [32] & 2020 & $\mathrm{O}$ & $\mathrm{O}$ & $\mathrm{O}$ & $\mathrm{O}$ & $\mathrm{O}$ & $\mathrm{O}$ & $\mathrm{O}$ & $\mathrm{O}$ & $\mathrm{O}$ \\
\hline
\end{tabular}

The statistically significant determinants of HB are shown in Figure 2. For the individual factors, there were significant variables in the demographic categories: age, gender, education, income, health insurance, race/ethnicity, and job. Among health functions, there were significant variables: sensory impairment, mobility impairment, disability, and activities of daily living/instrumental activities of daily living (ADL/IADL) dependency. In addition, irregular exercise and poor intake were significant in the health behavior category. In the physical category, weight loss, obesity, sarcopenia, oral health, and low limb pain were significant. In addition, there were significant variables: depression, fear of falling, anxiety, loneliness, and sense of coherence. Social support, social roles, and the community were significant social factors. For environmental factors, stairs, heavy doors, and land use were significant variables. 


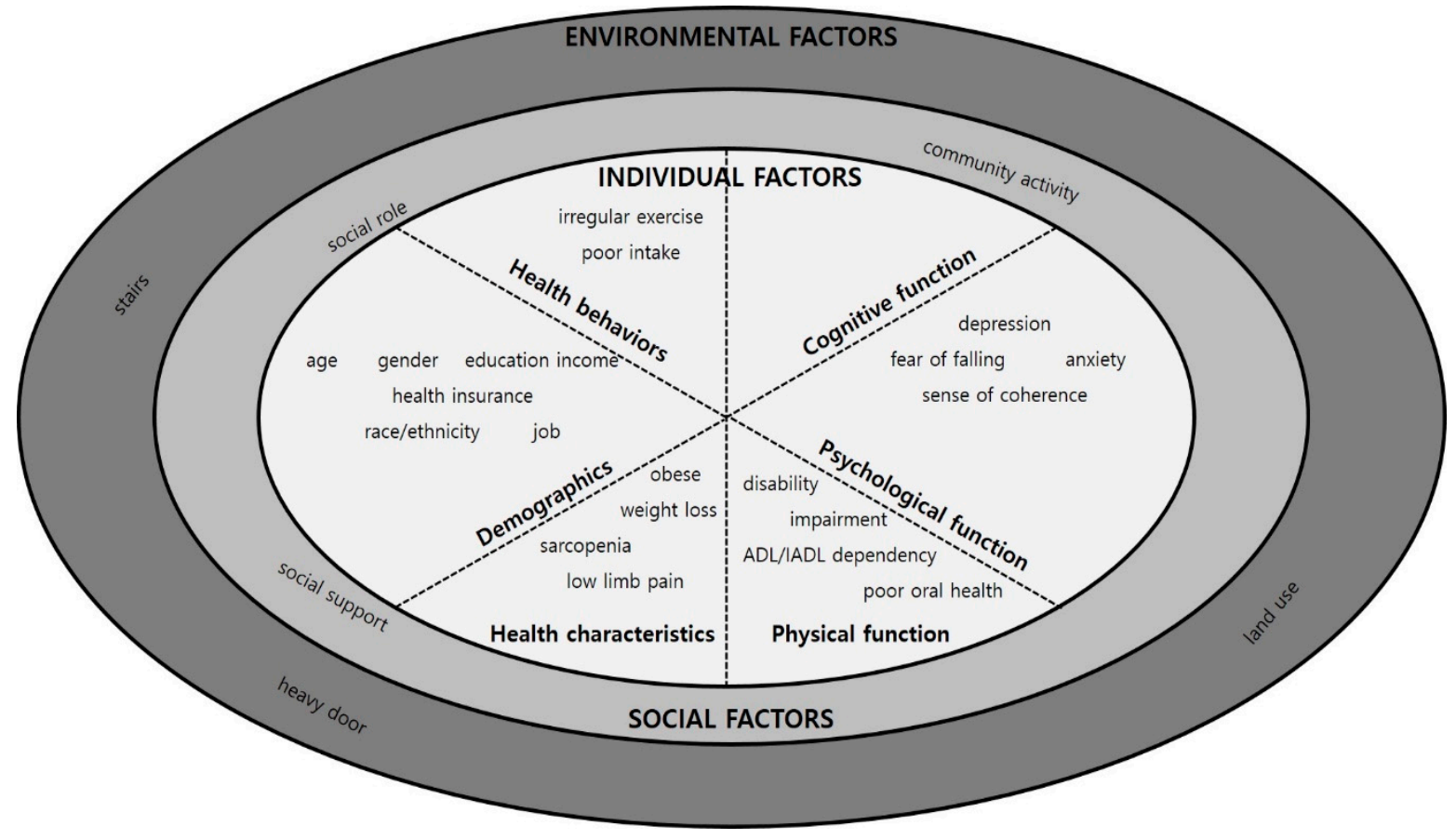

Figure 2. Significant determinants of $\mathrm{HB}$.

\section{Discussion}

This study identified the definition, measurement, and determinants of HB through a scoping review. We discuss these points and suggest their implications.

First, as a result of the scoping review, we found two definitions of HB to be widely used in the studies: "confined to home or remain inside the home" and "infrequently going outside the house." The former, home confinement or remaining inside the home, occurs due to a decline in an individual's ability to move, a medical problem that makes it difficult to move, and/or a lack of resources to help an individual who cannot move [17,21]. This definition was used in early studies $[9,17,18,41]$ and in studies defining targets for providing long-term care services in Japan [52] or Medicaid in the United States [17,21]. This definition is in line with the gerontological conceptual framework that the impact of late-life disability is based on the confluence of personal capacity and the ability of social support to compensate for limitations in capacity [53,54]. In contrast, "infrequently going outside the house" is presented as the result that reflects the individual's will and surrounding environment as well as their ability to move [47]. This definition is widely used in epidemiological studies [23], especially in Japan [2,29,38,49].

Through two HB definition analyses, we found that the common attribute of $\mathrm{HB}$ in previous studies is "the boundary of daily life is limited to home." Life-space mobility encompasses a person's independent mobility, requiring mobility-related physical activity (e.g., walking), and all movements supported by mobility aids and/or means of transportation [55]. Life-space mobility limited to home is similar to the common attributes of HB. Increasing living spaces would promote more activities and increase people's wellbeing [56]. Therefore, it is important to understand the meaning and boundaries of older adults' daily lives to prevent $\mathrm{HB}$.

Second, to measure or assess $\mathrm{HB}$, a question about the frequency of going out was used in most epidemiological studies. However, the observation period for measurement, the definition of "going out," and the criteria for determining HB were different. For this reason, the prevalence of $\mathrm{HB}$ varies from 3.5\% [18] to 26.5\% [37]. Gilber et al. examined the validity of one question, but the cut-off was "never or almost never except for emergencies on a six-point scale of frequency (almost every day, a few times a week, once a week, several times a month, less than several times a month but more than just for emergencies, 
and never or almost never except for emergencies)" [12]. Although one study reported that rarely going out has a higher probability of mobility disability [57] and death [58] than going out daily, previous researchers used a variety of cutoffs for the frequency of going out, which is the criterion of $\mathrm{HB}[9,39,50]$.

Some researchers have classified HB, beginning with the idea that HB people may have different health effects depending on the characteristics, prognosis, and causes of HB. For example, some researchers categorized HB into three groups based on the availability of help for going out and the difficulty of going out [26]. Another researcher [38] considered social isolation to be an important aspect of $\mathrm{HB}$ and classified $\mathrm{HB}$ into four groups: immobility and social exchange. However, some researchers have recently viewed $\mathrm{HB}$ as a continuous and dynamic process. For example, Xiang et al. defined HB as a continuum of outdoor mobility determined by physical capacity, availability of social support, and degree of autonomy [32]. Meng et al. evaluated the severity of HB based on the frequency of social contact, frequency of going out, time spent at home, and the need for help to go out [39]. When HB status is assessed, it is very important to consider the meaning of $\mathrm{HB}$ and to recognize $\mathrm{HB}$ as a preventable and manageable health problem. In addition, rather than simply screening HB people who stay at home all the time, it is necessary to develop a multidimensional tool that screens high-risk groups and provides appropriate interventions to prevent HB. For HB status assessment and comparison between nations, it is necessary to be able to measure and compare $\mathrm{HB}$ prevalence rates using standardized measurements.

Finally, we regard $\mathrm{HB}$ as a health outcome and identify the influencing factors of $\mathrm{HB}$ in older adults based on the revised ecological model. The theoretical framework for explaining $\mathrm{HB}$ was also applied in line with the changes in the definition of $\mathrm{HB}$ and its determinants. In a previous study, a conceptual framework related to the aging or disability process was applied to explain HB [26]. However, recent researchers have tried to explain HB and its influencing factors by applying an ecological model [20] or the ICF model [32]. Although few studies have applied the conceptual framework to preceding factors and the consequences of $\mathrm{HB}$, it is interesting to focus on $\mathrm{HB}$ due to social issues or environmental factors.

As a result of classifying related variables using the revised ecological model, most researchers focused on individual factors, including health characteristics, physical function, and psychological function. Early researchers considered individual factors such as immobility or medical conditions, psychological issues, and decreased autonomy as the causes of HB. In addition, physical function and physical illness should be managed to prevent $\mathrm{HB}$ in older adults because HB occurs due to physical limitations or lack of social support for going out. However, recent studies have reported that $\mathrm{HB}$ occurs due to not only individual factors, but also social factors $[2,18,19,23,27,29-32,44]$ or environmental and geographic characteristics $[6,18,29,32,47,50]$. Therefore, it is necessary to identify the various factors that influence HB by applying a theoretical framework.

Our findings have important implications for the expansion of the current view of HB research. We suggest that determinant survey is based on a theoretical model, especially from social and environmental perspectives. Individual factors have already been studied, but social and environmental factors have not been studied. The effects of various social factors such as social role, community participation, social networks, social isolation, and social capital on HB need to be identified.

As a result of this scoping review, social role, social support, and community participation among social factors have been identified as factors influencing HB status. In a previous study, HB increased loneliness and social isolation, which has been reported to worsen mental health among HB older adults [20,59]. Given that both social isolation and $\mathrm{HB}$ are strongly related to an increased risk of mortality, the coexistence of social isolation and HB may increase the risk of mortality as a synergistic effect [6]. Therefore, to prevent or manage $\mathrm{HB}$, it is necessary to recognize the importance of establishing a support system to maintain social relationships and participation and develop formal social support services to help people go out regularly. 
In South Korea, long-term care recipients mainly use housekeeping and living support services at home among the care services offered by the Korea Long-Term Care Insurance system [60]. In contrast, since April 2006 in Japan, people with HB have been specified as eligible for public long-term care insurance (LTCI), and LTCI has provided nursing and care services for HB people, as well as HB prevention services [16]. Therefore, there is a need to develop a service to prevent $\mathrm{HB}$ in older adults and provide proper home-based primary services for $\mathrm{HB}$ adults in long-term care services.

Recent studies have considered environmental factors, including the physical environment in the house, the residential area, the availability of transportation facilities, and the perception of neighborhood environment as influencing factors of HB. The scoping review showed that, among the environmental factors, neighborhood environment and physical obstacles to leaving the house, such as heavy doors and the presence of stairs, were significant factors influencing HB $[29,50]$. Therefore, to prevent $\mathrm{HB}$, it is necessary to support environmental improvement projects in homes where older adults reside. In addition, communities with extensive transportation networks and walking access to various non-residential properties are ideal for encouraging the elderly to go outside their homes.

This study has several limitations. First, we set the inclusion criteria to focus on the general HB. HB-related disabilities and diseases, such as mental health problems, were excluded from the study. This was done to focus on older people's HB, but in order to identify $\mathrm{HB}$ as a whole, it is necessary to expand the inclusion criteria in further research. Second, the perspective regarding $\mathrm{HB}$ has changed; so, it is important to recognize that this review represents a slice of information at one point in time. Third, we included only English-language abstracts. Papers initially written in Japanese were not included in this review. In addition, we did not include gray literature or doctoral theses. Finally, we conducted a scoping review. Therefore, we did not evaluate the quality of paper, publication type, etc., and we excluded papers based on their quality.

\section{Conclusions}

This study was conducted to identify the definition and determinants of $\mathrm{HB}$ and its recent trends through a scoping review. The definition and classification of HB is constantly changing and expanding as a result of changing perspectives on the determinants of HB. Initially, HB was defined to clarify the specific service target, but its definition and classification gradually changed as HB came to be regarded as a health problem that needs to be prevented and managed. Based on the results of this study, we provide the following suggestions and implications. First, it is necessary to develop a multidimensional tool to measure $\mathrm{HB}$ so that it cannot only screen individuals at risk of becoming $\mathrm{HB}$, but also suggest interventions for preventing and managing HB. Prior to this, an in-depth study should be conducted on the meaning and pattern of the boundary of daily life for older people in a cultural context. Second, the factors influencing HB, particularly individual factors, have been studied. The importance of environmental and social factors is emerging as an important determinant of HB. Therefore, it is necessary to identify the various factors that affect HB by applying a theoretical framework. Finally, as society ages, an increase in the number of older people who need long-term care, including HB individuals, has emerged as a major social problem. Living in "the boundary of daily life is limited to home" will negatively affect older adults' health and decrease their quality of life, while incurring enormous social costs to provide older adults with adequate services. Therefore, there is an urgent need to develop health policies to prevent $\mathrm{HB}$ in older adults.

Author Contributions: Y.K. and W.N. conceived and designed the study; Y.K. and W.N. analyzed the data; all authors wrote the first draft and critical discussion. Both authors have read and agreed to the published version of the manuscript.

Funding: This research received no external funding. 
Institutional Review Board Statement: The study was conducted in accordance with the guidelines of the Declaration of Helsinki and approved by the Institutional Review Board of Gachon University (IRB no. 1044396-202003-HR-066-01).

Informed Consent Statement: Not applicable.

Data Availability Statement: The data that support the findings of this study are available from the corresponding author upon reasonable request.

Conflicts of Interest: The authors have no conflicts of interest to declare.

\section{References}

1. World Health Organization. Available online: https://www.who.int/health-topics/ageing\#tab=tab_1 (accessed on 15 January 2021).

2. Uemura, K.; Makizako, H.; Lee, S.; Doi, T.; Lee, S.; Tsutsumimoto, K.; Shimada, H. The impact of sarcopenia on incident homebound status among community-dwelling older adults: A prospective cohort study. Maturitas 2018, 113, 26-31. [CrossRef]

3. Qiu, W.Q.; Dean, M.; Liu, T.; George, L.; Gann, M.; Cohen, J.; Bruce, M.L. Physical and mental health of homebound older adults: An overlooked population. J. Am. Geriatr. Soc. 2010, 58, 2423-2428. [CrossRef]

4. Ramraj, B.; Logaraj, M. Is home bound a major burden towards health access among the elderly population? A community based cross sectional study in the selected northern districts in Tamilnadu. Clin. Epidemiol. Glob. Health 2021, 9, 132-136. [CrossRef]

5. Musich, S.; Wang, S.S.; Hawkins, K.; Yeh, C.S. Homebound older adults: Prevalence, characteristics, health care utilization and quality of care. Geriatr. Nurs. 2015, 36, 445-450. [CrossRef] [PubMed]

6. $\quad$ Sakurai, R.; Yasunaga, M.; Nishi, M.; Fukaya, T.; Hasebe, M.; Murayama, Y.; Koike, T.; Matsunaga, H.; Nonaka, K.; Suzuki, H.; et al. Co-existence of social isolation and homebound status increase the risk of all-cause mortality. Int. Psychogeriatr. 2019, 31, 703-711. [CrossRef] [PubMed]

7. Donovan, N.J. Timely Insights into the Treatment of Social Disconnection in Lonely, Homebound Older Adults. Am. J. Geriatr. Psychiatry 2020, 28, 709-711. [CrossRef]

8. Cohen-Mansfield, J.; Shmotkin, D.; Hazan, H. The effect of homebound status on older persons. J. Am. Geriatr. Soc. 2010, 58, 2358-2362. [CrossRef] [PubMed]

9. Herr, M.; Latouche, A.; Ankri, J. Homebound status increases death risk within two years in the elderly: Results from a national longitudinal survey. Arch. Gerontol. Geriatr. 2013, 56, 258-264. [CrossRef] [PubMed]

10. Hamazaki, Y.; Morikawa, Y.; Morimoto, S.; Nakagawa, H. Difference in the impact of homebound status on functional decline between independent older men and women: A 2 year follow-up study. Jpn. J. Nurs. Sci. 2016, 13, 265-275. [CrossRef]

11. Lee, J.; Suh, Y.; Kim, Y. Multidimensional Factors Affecting Homebound Older Adults: A Systematic Review. SSRN 2020. [CrossRef]

12. Gilbert, G.H.; Branch, L.G.; Orav, E.J. An operational definition of the homebound. Health Serv. Res. 1992, 26, 787-800.

13. Ageing Planning Office. Write Up on Singapore's Long-Term Care System. In Proceedings of the 12th ASEAN and Japan High Level Officials Meeting on Caring Societies, Tokyo, Japan, 21-23 October 2014. Available online: http://www.mhlw.go.jp/bunya/ kokusaigyomu/asean/2014/dl/Singapore-2_CountryReport.pdf (accessed on 15 December 2020).

14. Genet, N.; Boerma, W.G.; Kringos, D.S.; Bouman, A.; Francke, A.L.; Fagerström, C.; Melchiorre, M.G.; Greco, C.; Devillé, W. Home care in Europe: A systematic literature review. BMC Health Serv. Res. 2011, 11, 207. [CrossRef] [PubMed]

15. Malmberg, B.; Ernsth, M.; Larsson, B.; Zarit, S.H. Angels of the nights: Evening and night patrols for homebound elders in Sweden. Gerontologist 2003, 43, 761-765. [CrossRef] [PubMed]

16. Olivares-Tirado, P.; Tamiya, N.; Kashiwagi, M.; Kashiwagi, K. Predictors of the highest long-term care expenditures in Japan. BMC Health Serv. Res. 2011, 11, 103. [CrossRef] [PubMed]

17. Bruce, M.L.; McNamara, R. Psychiatric status among the homebound elderly: An epidemiologic perspective. J. Am. Geriatr. Soc. 1992, 40, 561-566. [CrossRef] [PubMed]

18. Lindesay, J.; Thompson, C. Housebound elderly people: Definition, prevalence and characteristics. Int. J. Geriatr. Psychiatry 1993, 8, 231-237. [CrossRef]

19. Ganguli, M.; Fox, A.; Gilby, J.; Belle, S. Characteristics of rural homebound older adults: A community-based study. J. Am. Geriatr. Soc. 1996, 44, 363-370. [CrossRef] [PubMed]

20. Xiang, X.; Chen, J.; Kim, M. Trajectories of Homebound Status in Medicare Beneficiaries Aged 65 and Older. Gerontologist 2020, 60, 101-111. [CrossRef]

21. Engberg, S.; Sereika, S.; Weber, E.; Engberg, R.; McDowell, B.J.; Reynolds, C.F. Prevalence and recognition of depressive symptoms among homebound older adults with urinary incontinence. J. Geriatr. Psychiatry Neurol. 2001, 14, 130-139. [CrossRef] [PubMed]

22. Ornstein, K.A.; Leff, B.; Covinsky, K.E.; Ritchie, C.S.; Federman, A.D.; Roberts, L.; Kelley, A.S.; Siu, A.L.; Szanton, S.L. Epidemiology of the homebound population in the United States. JAMA Intern. Med. 2015, 175, 1180-1186. [CrossRef] [PubMed]

23. Umegaki, H.; Yanagawa, M.; Nakashima, H.; Makino, T.; Kuzuya, M.; Hidetoshi, H. The prevalence of homebound individuals in the elderly population: A survey in a city area in Japan. Nagoya J. Med. Sci. 2015, 77, 439-446. [CrossRef]

24. Zhao, Y.L.; Alderden, J.; Lind, B.; Stibrany, J. Risk factors for falls in homebound community-dwelling older adults. Public Health Nurs. 2019, 36, 772-778. [CrossRef] [PubMed] 
25. Xiang, X.; Brooks, J. Correlates of depressive symptoms among homebound and semi-homebound older adults. J. Gerontol. Soc. Work 2017, 60, 201-214. [CrossRef]

26. Soones, T.; Federman, A.; Leff, B.; Siu, A.L.; Ornstein, K. Two-year mortality in homebound older adults: An analysis of the National Health and Aging Trends Study. J. Am. Geriatr. Soc. 2017, 65, 123-129. [CrossRef]

27. Jing, L.W.; Wang, F.L.; Zhang, X.L.; Yao, T.; Xing, F.M. Occurrence of and factors influencing elderly homebound in Chinese urban community: A cross-sectional study. Medicine 2017, 96, e7207. [CrossRef] [PubMed]

28. Choi, K.; Park, E.A.; Lee, I.S. Homebound status and related factors according to age in female elders in the community. J. Korean Acad. Nurs. 2012, 42, 291-301. [CrossRef]

29. Murayama, H.; Yoshie, S.; Sugawara, I.; Wakui, T.; Arami, R. Contextual effect of neighborhood environment on homebound elderly in a Japanese community. Arch. Gerontol. Geriatr. 2012, 54, 67-71. [CrossRef]

30. Negron-Blanco, L.; Pedro-Cuesta, J.; Almazan, J.; Rodriguez-Blazquez, C.; Franco, E.; Damian, J.; DISCAP-ARAGON Research Group. Prevalence of and factors associated with homebound status among adults in urban and rural Spanish populations. BMC Public Health 2016, 16, 574. [CrossRef]

31. Takahashi, K.; Kato, A.; Igari, T.; Sase, E.; Shibanuma, A.; Kikuchi, K.; Nanishi, K.; Jimba, M.; Yasuoka, J. Sense of coherence as a key to improve homebound status among older adults with urinary incontinence. Geriatr. Gerontol. Int. 2015, 15, 910-917. [CrossRef]

32. Xiang, X.; An, R.; Oh, H. The Bidirectional Relationship between Depressive Symptoms and Homebound Status among Older Adults. Article. J. Gerontol. B Psychol. Sci. Soc. Sci. 2020, 75, 357-366. [CrossRef]

33. Sallis, J.F.; Owen, N.; Fisher, E. Ecological models of health behavior. Health Behav. Theory Res. Pract. 2015, 5, 43-64.

34. Munn, Z.; Peters, M.D.J.; Stern, C.; Tufanaru, C.; McArthur, A.; Aromataris, E. Systematic review or scoping review? Guidance for authors when choosing between a systematic or scoping review approach. BMC Med. Res. Methodol. 2018, 18, 143. [CrossRef]

35. Sucharew, H.; Macaluso, M. Progress Notes: Methods for Research Evidence Synthesis: The Scoping Review Approach. J. Hosp. Med. 2019, 14, 416-418. [CrossRef] [PubMed]

36. Arksey, H.; O’Malley, L. Scoping studies: Towards a methodological framework. Int. J. Soc. Res. Methodol. 2005, 8, 19-32. [CrossRef]

37. Kono, A.; Kanagawa, K. Characteristics of housebound elderly by mobility level in Japan. Nurs. Health Sci. 2001, 3, 105-111. [CrossRef]

38. Kawamura, K.; Watanabe, M.; Watanabe, T.; Tanimoto, Y.; Matsuura, T.; Kono, K. Incidence of disability in housebound elderly people in a rural community in Japan. Geriatr. Gerontol. Int. 2005, 5, 234-241. [CrossRef]

39. Meng, L.; Liu, Y.; Feng, X.; Zhai, Y.; Liu, K. The mediating role of depression on the relationship between housebound status and cognitive function among the elderly in rural communities: A cross-sectional study. Arch. Gerontol. Geriatr. 2018, 78, 58-63. [CrossRef]

40. Inoue, K.; Matsumoto, M. Homebound status in a community-dwelling elderly population in Japan. Asia Pac. J. Public Health 2001, 13, 109-115. [CrossRef]

41. Kostanjsek, N. Use of the International Classification of Functioning, Disability and Health (ICF) as a conceptual framework and common language for disability statistics and health information systems. BMC Public Health 2011, 11 (Suppl. S4), S3. [CrossRef]

42. Sharkey, J.R.; Branch, L.G.; Zohoori, N.; Ciuliani, C.; Busby-Whitehead, B.; Haines, P.S. Inadequate nutrient intakes among homebound elderly and their correlation with individual characteristics and health-related factors. Am. J. Clin. Nutr. 2002, 76, 1435-1445. [CrossRef]

43. Choi, M.G.; McDougall, G.J. Comparison of depressive symptoms between homebound older adults and ambulatory older adults. Aging Ment. Health 2007, 11, 310-322. [CrossRef]

44. Katsumata, Y.; Arai, A.; Tamashiro, H. Contribution of falling and being homebound status to subsequent functional changes among the Japanese elderly living in a community. Arch. Gerontol. Geriatr. 2007, 45, 9-18. [CrossRef] [PubMed]

45. Locher, J.L.; Ritchie, C.S.; Robinson, C.O.; Roth, D.L.; Smith West, D.; Burgio, K.L. A multidimensional approach to understanding under-eating in homebound older adults: The importance of social factors. Gerontologist 2008, 48, 223-234. [CrossRef]

46. Cohen-Mansfiled, J.; Shomotkin, D.; Hazan, H. Homebound older persons: Prevalence, characteristics, and longitudinal predictors. Arch. Gerontol. Geriatr. 2012, 54, 55-60. [CrossRef]

47. Koyama, S.; Aida, J.; Kondo, K.; Yamamoto, T.; Saito, M.; Ohtsuka, R.; Nakade, M.; Osaka, K. Does poor dental health predict becoming homebound among older Japanese? BMC Oral Health 2016, 16, 51. [CrossRef] [PubMed]

48. Harada, K.; Lee, S.; Park, H.; Shimada, H.; Makizako, H.; Doi, T.; Yoshida, D.; Tsutsumimoto, K.; Anan, Y.; Uemura, K.; et al. Going outdoors and cognitive function among community-dwelling older adults: Moderating role of physical function. Geriatr. Gerontol. Int. 2016, 16, 65-73. [CrossRef]

49. De-Rosende Celeiro, I.; Santos-del-Riego, S.; Muñiz García, J. Homebound status among middle-aged and older adults with disabilities in ADLs and its associations with clinical, functional, and environmental factors. Disabil. Health J. 2017, 10, 145-151. [CrossRef]

50. Satariano, W. Epidemiology of Aging: An Ecological Approach; Jones \& Bartlett Learning: Burlington, MA, USA, 2006 ; pp. $39-78$.

51. Jensen, G.L.; Silver, H.J.; Roy, M.; Callahan, E.; Still, C.; Dupont, W. Obesity is a risk factor for reporting homebound status among community-dwelling older persons. Obesity 2006, 14, 509-517. [CrossRef]

52. Shinaki, S. Homeboundness in the elderly. Jpn. J. Geriatric. 2008, 45, 117-125. 
53. Freedman, V.A. Adopting the ICF language for studying late-life disability: A field of dreams? J. Gerontol. A Biol. Sci. Med. Sci. 2009, 64, 1172-1174. [CrossRef]

54. Freedman, V.A.; Kasper, J.D.; Spillman, B.C.; Agree, E.M.; Mor, V.; Wallace, R.B.; Wolf, D.A. Behavioral adaptation and late-life disability: A new spectrum for assessing public health impacts. Am. J. Public Health 2014, 104, e88-e94. [CrossRef] [PubMed]

55. Webber, S.C.; Porter, M.M.; Menec, V.H. Mobility in older adults: A comprehensive framework. Gerontologist 2010, 50, 443-450. [CrossRef] [PubMed]

56. Foye, C. The relationship between size of living space and subjective well-being. J. Happiness Stud. 2017, 18, 427-461. [CrossRef]

57. Fugita, K.; Fujiwara, Y.; Chaves, P.H.M.; Motohashi, Y.; Shinkai, S. Frequency of going outdoors as a good predictor for incident disability of physical function as well as disability recovery in community-dwelling older adults in rural Japan. J. Epodemiol. 2006, 16, 261-270. [CrossRef]

58. Jacocs, J.M.; Cohen, A.; Hammeman-Rozenberg, R.; Azoulay, D.; Maaravi, Y.; Stessman, J. Going outdoors daily predicts long-term functional and health benefits among ambulatory older people. J. Aging Health 2008, 20, 259.

59. Courtin, E.; Knapp, M. Social isolation, loneliness and health in old age: A scoping review. Health Soc. Care Community 2017, 25, 799-812. [CrossRef] [PubMed]

60. Ga, H. Long-term care system in Korea. Ann. Geriatr. Med. Res. 2020, 24, 181-186. [CrossRef] [PubMed] 